\title{
Determinación de la eficiencia del sistema de filtros de esponjas colgantes continuas (DHS) en el tratamiento de aguas residuales del centro Poblado de Marian - Huaraz (Perú)
}

\author{
DETERMINATION OF THE EFFICIENCY OF THE CONTINUOUS \\ HANGING SPONGE FILTERS SYSTEM (DHS) IN THE WASTEWATER \\ TREATMENT OF THE TOWN CENTER OF MARIAN - HUARAZ (PERU)
}

\author{
MARTÍN HUAMÁN CARRANZA§, YUDITH FLORES ALBORNOZ§, ROSARIO POLO \\ SALAZAR§, NINO ARAUJO JAMANCA§, KIKO DEPAZ CELI§ \\ Recibido: 05 agosto de 2019 / Aceptado: 16 setiembre de 2019
}

\author{
§scuela Profesional de Ingeniería Sanitaria, Facultad de Ciencias del Ambiente, Universidad \\ Nacional Santiago Antúnez de Mayolo - Perú
}

\begin{abstract}
Resumen
Las aguas residuales en zonas rurales se tratan generalmente mediante tanques sépticos por ser un sistema de baja complejo. La aplicación de reactores, con filtrantes como esponjas endurecidas (llamados también reactor DHS) fue desarrollada como un tratamiento secundario de efluente de un tanque séptico. Los medios filtrantes de los reactores han sido elaborados con esponjas de poliuretano de resina epóxica. Los sistemas se instalaron y se desarrollaron en la planta de tratamiento de aguas residuales de la localidad de Marian (Huaraz - Perú), donde se logró que el efluente del tanque séptico ingrese continuamente al reactor DHS. Los sistemas se desarrollaron a una temperatura que osciló de 11.25 a $15.30{ }^{\circ} \mathrm{C}$ y una concentración de $\mathrm{pH}$ de 7.3 a 8.11. Los reactores DHS mostraron eficiencia de remoción de contaminantes, se evaluó los porcentajes de remoción de la $\mathrm{DBO}_{5}$; y se observó que las esponjas G1, G3 y G6 obtuvieron la remoción del 50\%. Se verificó que la remoción de la materia orgánica fue lenta debido a la adaptación de los microorganismos. Evaluando los porcentajes de remoción de la DQO; se pudo observar que las esponjas G1, G3 y G6 mostraron remoción menor al 50\%, la mejor eficiencia que se obtuvo fue en la eliminación de los sólidos suspendidos totales (SST) con remoción cerca al 100\%, demostrando que las esponjas filtraron los SST. En la esponja G6 se observó la mayor eficiencia; evidencia que estos tratamientos permiten la oxidación de los compuestos nitrogenados generándose Nitrato, elemento nutritivo para el desarrollo de las plantas. Se concluye que los parámetros analizados han sido removidos eficientemente por el sistema DHS con esponjas endurecidas.

Palabras clave: tratamiento, efluente, eficiencia, esponjas
\end{abstract}

\begin{abstract}
Wastewater in rural areas is generally treated by septic tanks, because the system is the least complex. The application of reactors, with filters such as hardened sponges (also called DHS reactor) was developed as a secondary treatment of effluent from a septic tank. The filter media of the reactors have been made with epoxy resin polyurethane sponges. The systems were installed and developed at the sewage treatment plant in the town of Marian (Huaraz - Peru), where septic tank effluent was continuously entered into the DHS reactor. The systems were developed at a temperature that ranged from 11.25 to $15.30{ }^{\circ} \mathrm{C}$ and a $\mathrm{pH}$ concentration of 7.3 to 8.11. DHS reactors showed pollutant removal efficiencies, the removal percentages of BOD5 were evaluated; and it was observed that sponges G1, G3 and G6 obtained the removal of 50\%, then it was verified that the removal of organic matter was slow due to the adaptation of the microorganisms. Evaluating the COD removal percentages; it was observed that sponges G1, G3 and G6 showed removal percentage below $50 \%$, the best efficiency obtained was in the elimination of total suspended solids (SST) where there were high removal values close to $100 \%$, demonstrating that in all sponges solids are retained. Sponge G6 showed the highest generation efficiency, unlike sponges G1 and G2, which also have high values; which demonstrates that these treatments do allow the oxidation of nitrogen compounds, generating Nitrate, a nutritive element for plant development. It is concluded that the parameters analyzed have been efficiently removed by the DHS system with hardened sponges.

Keywords: Specific richness, diversity of species, ecosystem, lagoon.
\end{abstract}




\section{INTRODUCCIÓN}

La población de la sierra de Ancash, especialmente en las zonas rurales conoce, que los tratamientos básicos de las aguas residuales en las zonas rurales o en poblaciones pequeñas se realizan generalmente mediante tanques sépticos, debido a que el sistema es el menos complejo para el tratamiento de aguas residuales, así como en los costos de mantenimiento; sin embargo, este sistema no llega a cumplir con la remoción adecuada de los contaminantes presentes en las agua servidas domésticas, y principalmente tiene una baja de remoción los contaminantes microbiológicos y fisicoquímicos. Además, los efluentes de tanques sépticos no cumplen las normas nacionales de vertimiento de aguas residuales, los cuales, son los Límites Máximos Permisibles. Para mejorar el efluente de estos tipos de tratamiento se plantea implementar el sistema Downflow Hanging Sponge (DHS - filtros de esponjas colgantes continuas), el que tiene como meta la comparación de la capacidad de remoción de contaminantes fisicoquímicos y bacteriológicos en un medio de esponjas poliuretano con resina apóxico.

Con esta tecnología se propone una alternativa y tecnología para el tratamiento de aguas residuales domésticas en zonas rurales que tienen bajo costo de instalación, operación y mantenimiento de fácil uso para los operadores de los sistemas de saneamiento.

En el contexto de la situación descrita, se plantea el problema principal que consiste en buscar posibles soluciones con una alternativa para mejorar el efluente de estos tipos de tratamiento y plantear implementar el sistema DHS.

\section{MATERIALES Y MÉTODOS}

\section{TIPO Y DISEÑO DE LA INVESTIGACIÓN}

El tipo de la investigación fue descriptivo - cuantitativo; debido a que, consistió en la caracterización de un hecho, fenómeno, individuo o grupo, con el fin de establecer su estructura o comportamiento, y se busca especificar las propiedades importantes para medir y evaluar aspectos, dimensiones o componentes del fenómeno a estudiar.

El diseño de investigación es pre-experimental. Debido a que por medio de este tipo de investigación se puede aproximar a resultados de una investigación experimental en situaciones en las que no es posible el control y manipulación absolutos de las variables.

\section{RECOLECCIÓN DE LA INFORMACIÓN}

Se realizó siguiendo la metodología planteada, donde se desarrollaron todos los procedimientos adecuados para obtener el resultado final; se realizaron muestreos semanales y su análisis correspondiente de los parámetros en estudio en el sistema Downflow Hanging Sponge (DHS) de todos los tipos de esponja G1, G2, G3, G4, G5 y G6 implementado como post tratamiento del efluente del Tanque Séptico en la localidad de Marian, a través de instrumentos como el Multiparamétrico.

El cálculo del tiempo de retención hidráulica, dependió del diseño del material filtrante, 
y ésta a su vez, de la porosidad de la esponja el cual se investigó a través de ensayos de absorción que se llevó cabo en los Laboratorios de la UNASAM. Los resultados fueron anotados en formatos para reportes y registros propiamente elaborados.

Los parámetros: Solidos Totales en Suspensión, Aceites y Grasas, $\mathrm{DBO}_{5}, \mathrm{DQO}$, Coliformes Termotolerantes, N-Amoniacal y Nitratos, fueron analizados en el Laboratorio de Calidad Ambiental de la UNASAM.

El caudal, temperatura y $\mathrm{pH}$ fueron medidos en campo a través de un Datalogger. Los resultados se anotaron en formatos para reportes y registros propiamente elaborados.

El tiempo de investigación dependió de la adecuación y estabilidad de los microorganismos del sistema Downflow Hanging Sponge (DHS).

\section{UNIDAD DE ANÁLISIS Y MUESTRA}

Las aguas utilizadas corresponden a las generadas en la localidad de Marian (Ancash) y que son tratadas a través del sistema de tanque séptico. Para este estudio el caudal promedio obtenido de las aguas residuales generadas en la localidad de Marian fueron de $0.52 \mathrm{l} / \mathrm{s}$ calculado en campo mediante aforos durante 5 días consecutivos.

Se implementó el reactor DHS con diversas esponjas: G1, G2, G3 G4, G5 y G6, y se derivaron las aguas del tanque séptico que atraviesa el sedimentador piloto instalado.

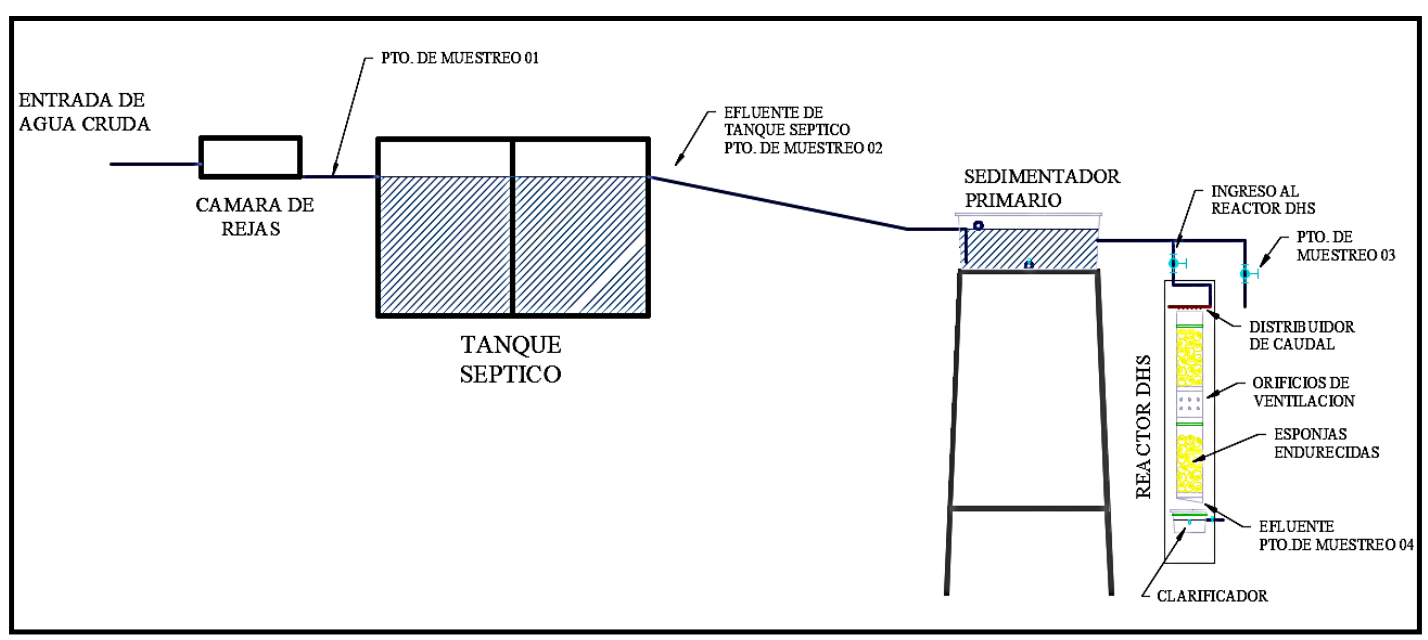

Figura 1: Esquema general del sistema de tratamiento mediante tanque séptico y sistema Down-flow Hanging Sponge

El desarrollo de esta investigación se basa en los principios de la degradación de la materia orgánica en un medio fijo, donde el medio para la adsorción de los microorganismos son las esponjas endurecidas. 


\section{PLAN DE PROCESAMIENTO Y ANÁLISIS ESTADÍSTICO DE LA INFORMACIÓN}

Para el análisis estadístico se empleó el Software SPSS versión 15.0. Se realizaron tablas de frecuencia para un análisis experimental. Las variables categóricas fueron comparadas con el Test de Fisher, la prueba de T - Student, fue empleada para evaluar significancia estadística de variables cuantitativas.

\section{VALIDEZ Y CONFIABILIDAD DE LA INFORMACIÓN}

La información que se obtiene de los análisis de laboratorio acreditado es válida y confiable, porque es representa las características reales de la fuente de agua evaluada para comprobar que si funcionan como ayudantes de coagulación y que se demuestran en los resultados presentados, validadas por el método científico.

\section{RESULTADOS Y DISCUSIÓN}

\section{CARACTERÍSTICAS DE LAS AGUAS RESIDUALES CRUDAS}

Las características fisicoquímicas y microbiológicas obtenidas al analizar el agua residual al ingreso de la planta de tratamiento, se muestran en la Tabla 1, en el que se indica todos los parámetros de interés para la investigación. Estos datos se han obtenido en el día 10 del inicio de la toma de datos del reactor piloto DHS G1, G2, G3, G4, G5 y G6.

Además, es necesario indicar que hubo 26 días de adaptación y adecuación. Se empezó con la toma de datos de temperatura y $\mathrm{pH}$ al primer día, posteriormente a los 10 días se obtuvo el primer muestreo para la caracterización de las aguas residuales de la planta de tratamiento existente.

Se observa que los resultados obtenidos, en $\mathrm{DBO}_{5}$ fue de $333 \mathrm{mg} / \mathrm{l}$ y DQO de $669 \mathrm{mg} / \mathrm{l}$; así mismo SST fue $140 \mathrm{mg} / \mathrm{l}$, y aceites y grasas $325 \mathrm{mg} / \mathrm{l}$; en caso de los nutrientes fue $10.6 \mathrm{mg} / \mathrm{l}$ de N-Amoniacal y 0.05 de Nitratos; finalmente el resultado de los Coliformes Fecales fue de 2.10 x107 NMP/100 ml.

Tabla 1. Características fisicoquímicas y microbiológicas del agua residual cruda.

\begin{tabular}{|c|c|c|}
\hline PARÁMETRO & UNIDAD & $\begin{array}{l}\text { AA. Residual } \\
\text { Cruda - C.rejas }\end{array}$ \\
\hline Potencial de Hidrogeno $(\mathrm{pH})$ & Unidad de $\mathrm{pH}$ & 6.32 \\
\hline Temperatura & ${ }^{\circ} \mathrm{C}$ & 12.8 \\
\hline Aceites y Grasas & $\mathrm{mg} / \mathrm{l}$ & 325.00 \\
\hline Sólidos Totales en Suspensión & $\mathrm{mg} / \mathrm{l}$ & 140.00 \\
\hline N-Amoniacal & $\mathrm{mg} / 1 \mathrm{NO}_{4}-\mathrm{N}$ & 10.60 \\
\hline Nitratos & $\mathrm{mg} / 1 \mathrm{NO}_{3}$ & 0.05 \\
\hline Demanda Bioquímica de Oxigeno & $\mathrm{mg} / \mathrm{DBO}_{5}$ & 333.00 \\
\hline Demanda Química de Oxigeno & mg/l DQO & 669.00 \\
\hline Coliformes Fecales & $\mathrm{NMP} / 100 \mathrm{ml}$ & $2.40 \times 107$ \\
\hline
\end{tabular}


El caudal se aforó en intervalos de cada hora, para así obtener el caudal horario, donde se obtuvo como el caudal promedio diario $Q p=0.52$ 1/s de producción de agua residual que llega a la planta de tratamiento de aguas residuales de la localidad de Marian; así mismo, el caudal máximo horario obtenido fue $\mathrm{Qmax}=1.07 \mathrm{l} / \mathrm{s}$ y el caudal mínimo horario fue Qmin $=0.251 / \mathrm{s}$ (Figura 2).

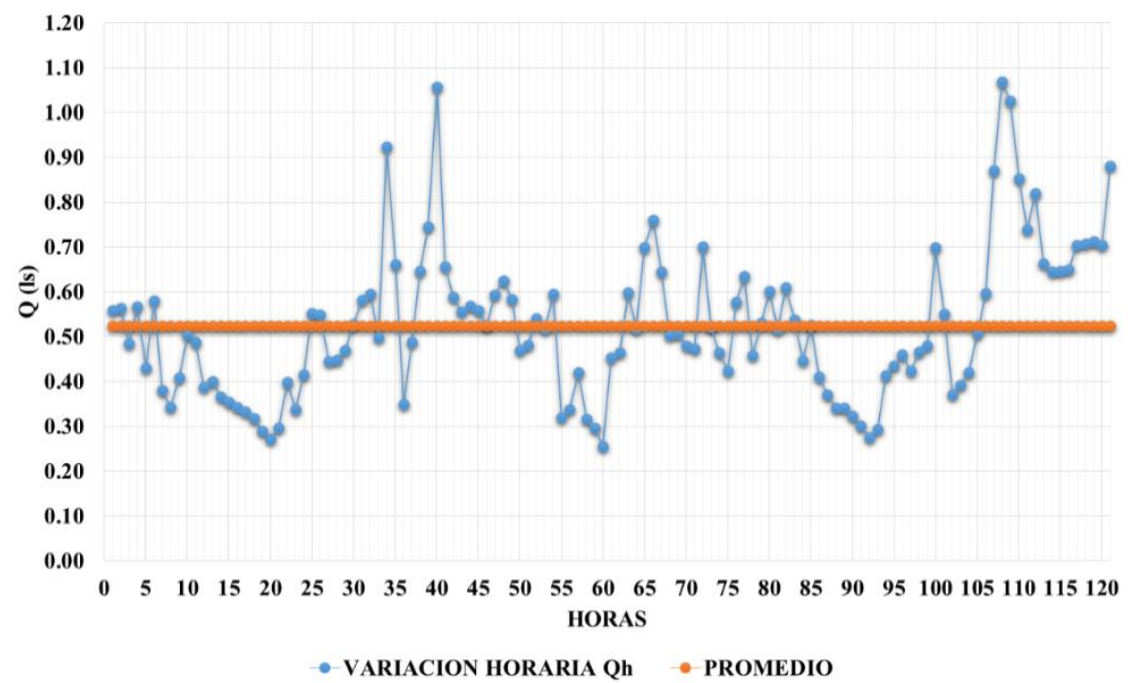

Figura 2: medición de caudal durante la investigación

Evaluando los porcentajes de remoción de la $\mathrm{DBO}_{5}$ de las diversas esponjas; se observó que las esponjas G1, G3 y G6 removieron casi el 50\%, evidenciando remoción de materia orgánica; en cuanto a las esponjas G2, G4 y G5, los porcentajes de remoción fueron bajos debido a la forma de la esponja y la lenta adaptación de los microorganismos, dificultando la remoción de la materia orgánica (Figura 3).

La materia orgánica fue removida activamente por parte de los microorganismos; salvo algunos puntos elevados debido a la saturación de la materia orgánica en la esponja.

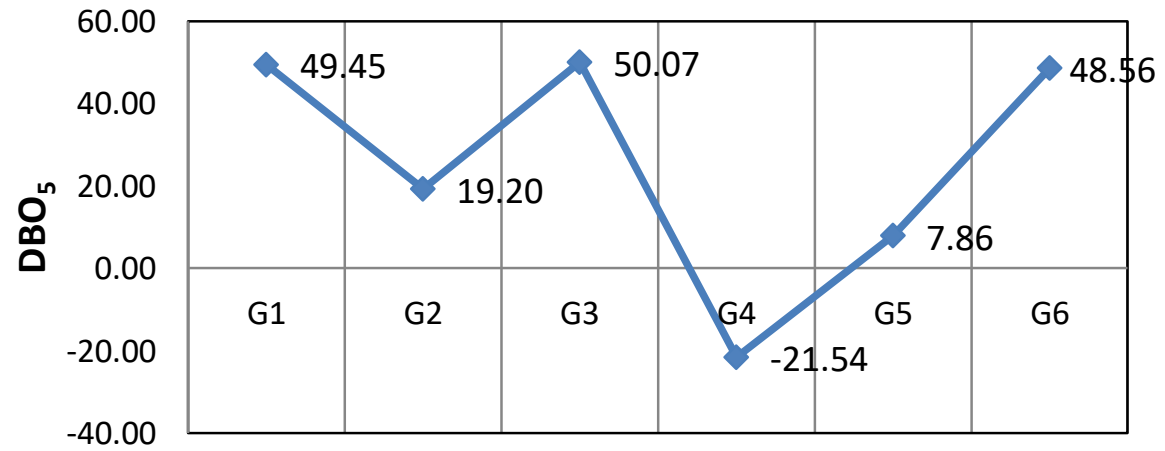

Figura 3: Eficiencia de remoción de $\mathrm{DBO}_{5}$ en los reactores DHS

Evaluando los porcentajes de remoción de la DQO; se observó que las esponjas G1, G3 y G6 removieron menos del 50\%, evidenciando que no hubo una remoción eficiente de la materia orgánica; en cuanto a las esponjas G2, G4 y G5 muestran porcentajes bajos debido a que las reacciones químicas se desarrollan de forma lenta, dificultando la remoción de la materia orgánica (Figura 4). 


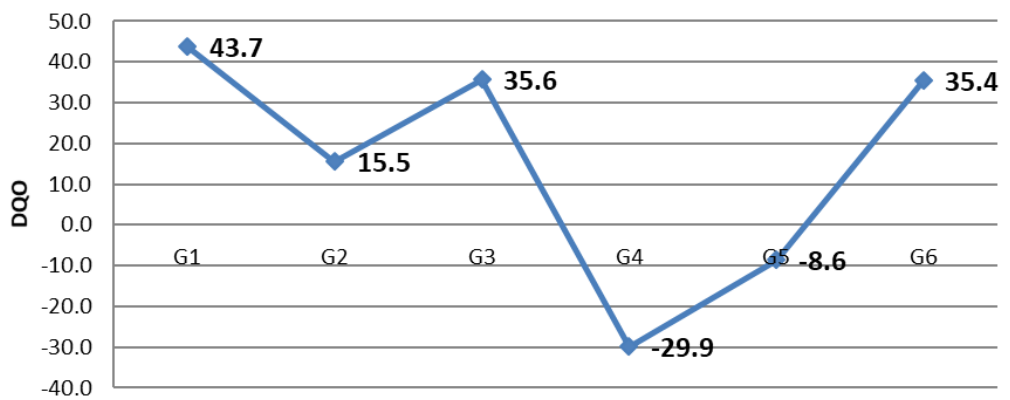

Figura 4: Eficiencia de remoción de DQO en los reactores DHS

Evaluando los porcentajes de remoción de la Coliformes termotolerantes de las diversas esponjas; se observó que las esponjas G1 y G3 removieron cerca al 50\%; en cuanto a las esponjas G2, G4, G5 y G6, la remoción fue baja (Figura 5).

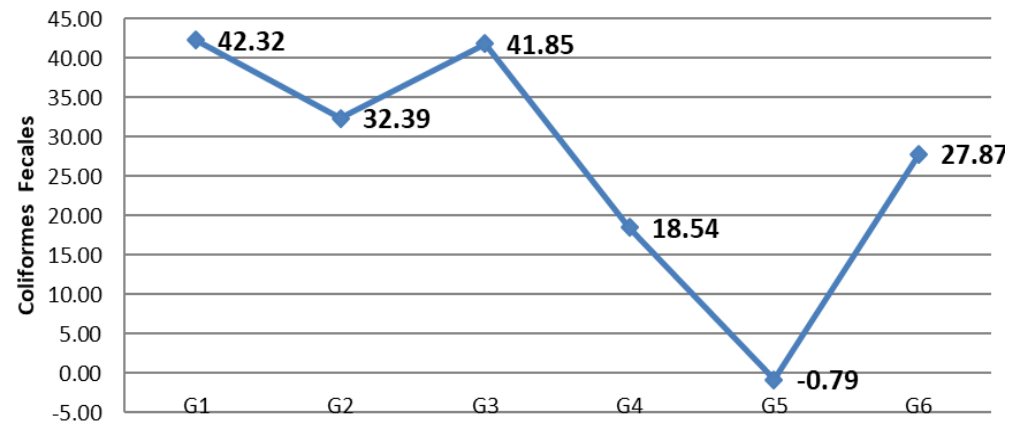

Figura 5: Eficiencia de remoción de Coliformes termotolerantes en los reactores DHS

En cuanto a la eficiencia de remoción de los SST; estos muestran valores altos de remoción cerca al 100\%, demostrando que en todas las esponjas los sólidos quedan retenidos (Figura 6).

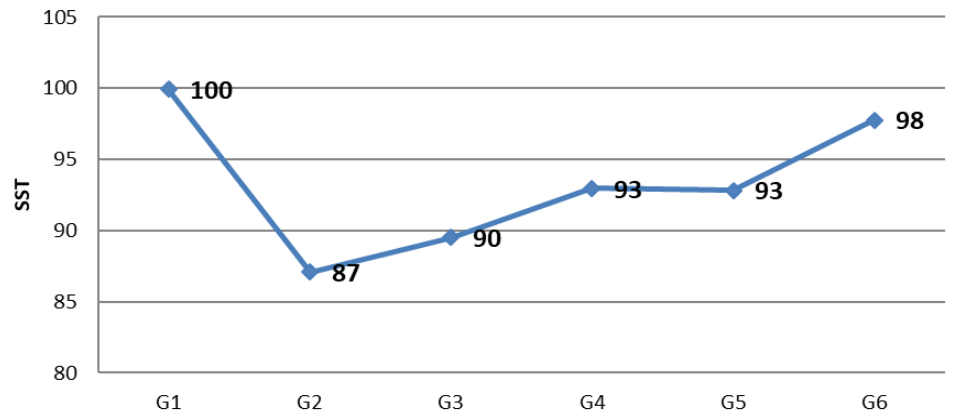

Figura 6: Eficiencia de remoción de SST en los reactores DHS

En cuanto a la eficiencia de remoción de los Aceites y Grasas; estos muestran valores altos de remoción cerca al 100\%, demostrando que en todas las esponjas los sólidos quedan retenidos (Figura 7). 


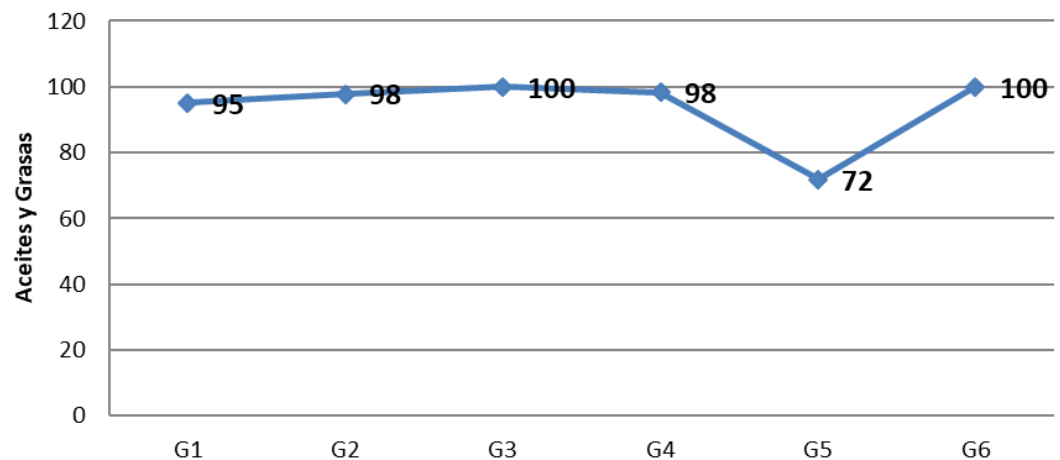

Figura 7: Eficiencia de remoción de Aceites y grasas en los reactores DHS

En cuanto a la remoción del N-Amoniacal; se puede observar que la esponja G2 muestra una remoción de 18\%, mientras que la esponja G5 y G6 muestran una remoción negativa, lo que demuestra que la esponja G2 retiene el N-Amoniacal mientras que la esponja G5 y G6 no remueven el N-Amoniacal

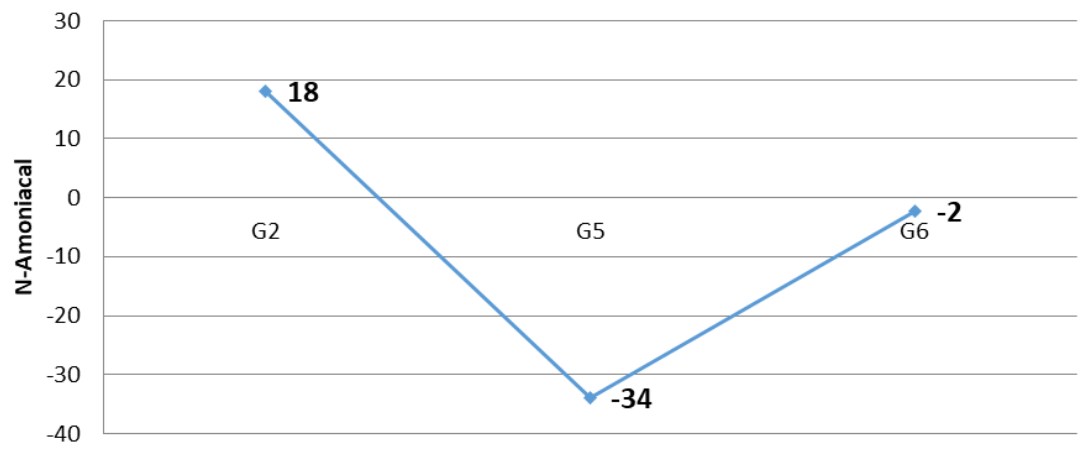

Figura 8: Eficiencia de remoción de N-Amoniacal en los reactores DHS

En cuanto al porcentaje de generación de $\mathrm{NO}_{3}$ en las esponjas evaluadas; se puede observar que la esponja G6 muestra la mayor eficiencia de generación a diferencia de la esponja G1 y G2 que también presentan valores altos; lo que demuestra que estos tratamientos si permiten la oxidación de los compuesto nitrogenados generándose Nitrato, elemento nutritivo para el desarrollo de las plantas

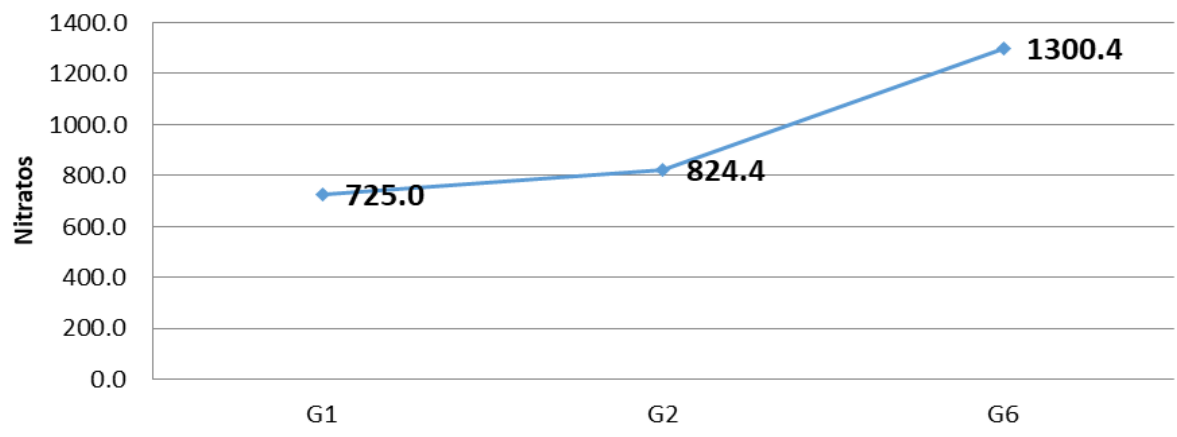

Figura 9: Eficiencia de remoción de Nitratos $\left(\mathrm{NO}_{3}\right)$ en los reactores DHS 


\section{DISCUSIÓN}

Los resultados obtenidos de los análisis realizados en los puntos de muestreos establecidos se compararan con los Estándares Nacionales e Internacionales para ello sólo se considerando los parámetros contemplados en ellos, teniendo en cuenta los valores mínimos de las normas, utilizando el agua tratada en el riego de plantas, uso agrícola y bebida para animales.

Estándares Nacionales: Los estándares de comparación adoptados son referenciales y se usan para realizar comparaciones con las condiciones naturales existentes en la zona, para ello se aplicará los Estándares de Calidad Ambiental (ECA) para Agua - Categoría 3: Riego de Vegetales y bebida de animales, Decreto Supremo Nº 004-.2017-MINAM.

Tabla 2. Estándares de Calidad Ambiental - Categoría 3

\begin{tabular}{|c|c|c|c|c|c|}
\hline \multirow[b]{2}{*}{ Parámetros } & \multirow{2}{*}{$\begin{array}{l}\text { Unidad de } \\
\text { medida }\end{array}$} & \multicolumn{3}{|c|}{ D1: Riego de vegetales } & \multirow{2}{*}{$\begin{array}{l}\text { D2: Bebida de } \\
\text { animales }\end{array}$} \\
\hline & & $\begin{array}{l}\text { Agua para riego no } \\
\text { restringido }\end{array}$ & & $\begin{array}{l}\text { Agua para riego } \\
\text { restringido }\end{array}$ & \\
\hline \multicolumn{6}{|l|}{ FISICOS Y QUIMICOS } \\
\hline $\begin{array}{l}\text { Demanda Bioquímica de } \\
\text { Oxígeno (DBO) }\end{array}$ & $\mathrm{mg} / \mathrm{l}$ & & 15 & & 15 \\
\hline $\begin{array}{l}\text { Demanda Química de } \\
\text { Oxígeno (DQO) }\end{array}$ & $\mathrm{mg} / \mathrm{l}$ & & 40 & & 40 \\
\hline Nitratos $\left(\mathrm{NO}_{3}\right)$ & $\mathrm{mg} / \mathrm{l}$ & & 0.1 & & 0.1 \\
\hline Aceites y Grasas & $\mathrm{mg} / \mathrm{l}$ & & 5 & & 10 \\
\hline N-Amoniacal & $\mathrm{mg} / \mathrm{l} /$ & & 1 & & 1 \\
\hline \multicolumn{6}{|l|}{ MICROBIOLÓGICOS } \\
\hline Coliformes Totales & $\mathrm{NMP} / 100 \mathrm{ml}$ & 1000 & & 2000 & 1000 \\
\hline Coliformes Termotolerantes & $\mathrm{NMP} / 100 \mathrm{ml}$ & 1000 & & 2000 & 1000 \\
\hline
\end{tabular}

Fuente: Estándares de Calidad Ambiental para Agua - D. S. N 004-2017-MINAM.

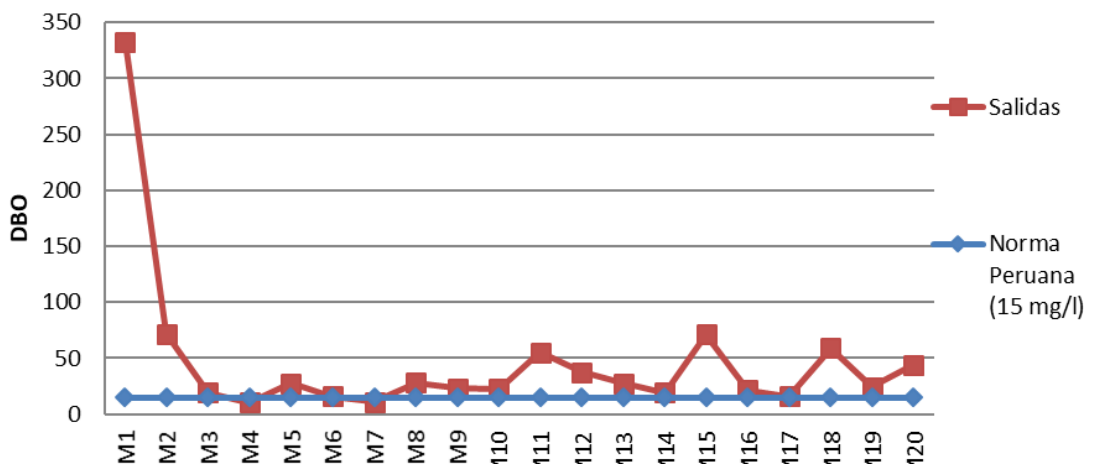

Figura 10. Remoción total de materia orgánica en los reactores DHS - G5

En cuanto a la remoción de la $\mathrm{DBO}_{5}$ en la salida de la esponja G5, se puede observar que la mayoría de los valores están cerca al valor límite permisible de la norma; pero al igual que los demás se requiere aplicar otros tratamientos para poder disminuir la materia orgánica antes de ser destinada el agua para algún uso.

En cuanto a la remoción de Aceites y Grasas en la salida de la esponja G6, se puede observar que los valores obtenidos están por debajo del límite permisible de la norma; lo que evidencia que el tratamiento con esponja si retiene los aceites y grasas.

En cuanto a la remoción de SST en la salida de la esponja G5, se puede observar que la 
mayoría de los valores están por debajo del límite permisible de la norma chilena y unos pocos están por cerca del límite permisible de la norma; lo que evidencia que el tratamiento con esponja si retiene los sólidos para su degradación mediante los microorganismos presentes.

\section{CONCLUSIONES}

En el presente trabajo de investigación se concluyó que la eficiencia de remoción de los parámetros evaluados en cada tipo de esponjas fue diferente; debido a, la forma y disposición en las cuales se colocan en el filtro.

Se determinó que el caudal, $\mathrm{pH}$ y temperatura son parámetros que influyen de manera directa en la adaptación y desarrollo de los microorganismos en las diferentes generaciones de esponjas.

En la evaluación del porcentaje de remoción de la $\mathrm{DBO}_{5}$ se puede concluir que las esponjas G1, G3 y G6 presentaron valores de $49.45 \%, 50.07 \%$ y $48.56 \%$ de remoción respectivamente aceptables en la remoción de la materia orgánica; mientras que la esponja G2, G4 y G5 presentan porcentajes bajos evidenciando su lenta degradación.

La evaluación del porcentaje de remoción de la DQO demostró que la esponjas G1, G3 y G6 presentaron valores de $43.70 \%, 35.60 \%$ y $35.40 \%$ de remoción respectivamente, lo que muestra porcentajes menores al 50\% de remoción; mientras que las esponjas G2, G4 y G5 presentaron porcentajes bajos evidenciando su lenta remoción.

La evaluación del porcentaje de remoción de Coliformes Fecales demostró que las esponjas G1, G2, G3 y G6 presentaron valores de $42.32 \%, 32.39 \%, 41.85 \%$ y $27.87 \%$ de remoción respectivamente, demostrando porcentajes menores al 50\%; mientras que las esponjas G4 y G5 presentan porcentajes bajos evidenciando su poca remoción.

En cuanto al porcentaje de remoción de los SST se concluyó que todas las esponjas presentan porcentajes de remoción cerca al 100\%, siendo el G1 el más eficiente mostrando un $100 \%$ de remoción.

En cuanto al porcentaje de remoción de los Aceites y Grasas se concluyó que todas las esponjas presentan porcentajes de remoción cerca al 100\%, siendo el G3 y G6 el más eficiente mostrando un $100 \%$ de remoción.

El porcentaje de remoción del N-Amoniacal se concluyó que la esponja G2 presentó una remoción de 18\%, mientras que las esponjas G5 y G6 mostraron porcentajes bajos, lo que evidencia su poca retención en la esponja.

En cuanto al porcentaje de generación del Nitrato se concluyó que las esponjas G1, G2 y G6 presentaron porcentajes altos de generación de Nitrato, siendo la esponja G6 la que presentó una producción mayor (1300.4\% de Nitrato).

De acuerdo a la Norma Peruana - ECA, el agua que salió de las esponjas G1, G2, G3, G4 y G6 presentaron $\mathrm{DBO}_{5}$ variables, cuyos valores se encontraron en la mayoría de los casos por encima de la norma, mientras que la esponja G5 mostró valores cerca al valor límite permisible de la norma. El agua que pasó por las esponjas G1, G2, G3, G4 y G6 
presentaron valores de DQO por encima del valor límite permisible de la norma, mientras que la esponja G5 presentó valores próximos al valor límite de la norma.

De acuerdo a la Norma Peruana - ECA, el agua que salió de las esponjas G1, G2, G3, G4, G5 y G6 presentó valores de Coliformes Fecales por encima del valor límite permisible de la norma.

En cuanto a los SST, se concluyó que todos los resultados obtenidos en cada una de las esponjas G1, G2, G3, G4, G5 y G6 estuvieron por debajo del límite permisible de la norma.

Con respecto a los Aceites y Grasas se puede concluir que al inicio algunos resultados estuvieron por encima del límite permisible de la norma, como es el caso de las esponjas G1, G2 y G5; pero posteriormente, los resultados están por debajo de límite permisible de la norma peruana.

El agua que sale de las esponjas G2, G5 y G6 presentan valores de N-Amoniacal por encima del valor límite permisible de la norma Peruana - ECA, el agua que sale de las esponjas G1, G2 y G6 presentan valores de Nitrato por encima del valor límite permisible de la norma.

\section{Referencias}

Aguamarket (2000). www.aguamarket.com/diccionario/terminos.asp. (Consultado el 28 de Diciembre del 2017)

American Public Health Association (APHA), American Water Works Association (AWWA) Y Water Polution Control Federation (WPCF). (1992). "Métodos normalizados para el análisis de aguas potables y residuales". Madrid - España. Ediciones Díaz de Santos S.A. 17 Edición APHA - AWWA - WPCF.

Decreto Supremo N 004-2017-MINAM .- “Aprueban Estándares de Calidad Ambiental (ECA) para Agua y establecen Disposiciones Complementarias”. http://www.minam.gob.pe/wpcontent/uploads/2017/06/DS-004-2017-MINAM.pdf. (Consultado el 6 de Setiembre del 2017)

Espinoza, A. (2017); Entrevista de Martín Huamán. "Historia de la ciudad de Huaraz". Huaraz Perú.

Esqueche, C. (2013), “Contactores Biológicos Rotativos". https://carlosesquecheangeles. wordpress.com/tag/residuos-solidos/ (Consultado el 15 de Diciembre del 2017)

Harada, H. (2001) "Nueva tecnología para el tratamiento secundario de los efluentes del reactor UASB”, University of Technology: Nagaoka, Japón.

Harada, H. (2008) "India-Japan International Collaboration for an Innovative Sewage Treatment Technology with Cost-effective and Minimum Energy Requirement", Asian Science and Technology Seminar (ASTS) in Thailand,

Hernandez, R., Fernández, C., Baptista, P. (2010), "Metodología de la Investigación”. México: Mc Graw Hill.

Marsilla, A. (2005) "Tratamiento de Aguas Residuales". http://www.tierramor.org/ Articulos/tratagua.htm (Consultado el 28 de Diciembre del 2017) 
Metcalf E. (1995), "Ingeniería de Aguas Residuales: Tratamiento , Vertido y Reutilización". Volumen 1, 3ra. Edición - Editorial: S.A. MCGRAW-HILL - INTERAMERICANA DE ESPAÑA.

Rosales, E. (2005). "Tanques Sépticos: Conceptos Teóricos Base y Aplicaciones”. Tomado de : https:/fibromuebles.com/userfiles/file/PRESENTACIONES/ Tanques_Septicos_LIBRO.pdf.

Steven, H., Diana, L. (2012). "Estudio del sistema Downflow Hanging Sponge (DHS) tipo bloques prismáticos en el tratamiento del efluente de un reactor anaerobio de manto de lodos y flujo ascendente". Universidad Nacional de Ingeniería. Lima, Perú.

Tandukar M. et al. (2005). “A Low-Cost Municipal Sewage Treatment System with a Combination of UASB and the "Fourth Generation" Down-Flow Hanging Sponge (DHS). Water Science \& Technology”. India.

Tawfik, A. (2008). "Sewage treatment using an integrated system consisting of anaerobic hybrid reactor (AHR) and downflow hanging sponge (DHS)" This research was supported in part by research grants from the Ministry of Education, Culture, Sports, Science and Technology; Japan, the Japan Society for the Promotion of Science, science and Technology Research partnership for Sustainable Development (SATREPS). 